\title{
On the Efficiency for Non-Endoreversible Stirling and Ericsson Cycles
}

\author{
Delfino Ladino-Luna, Pedro Portillo-Díaz, Ricardo T. Páez-Hernández \\ Universidad Autónoma Metropolitana-Azcapotzalco, Física de Procesos Irreversibles, México D.F., México \\ Email: d1l@correo.azc.uam.mx, pportillodaz@gmail.com,phrt@correo.azc.uam.mx
}

Received October 1, 2013; revised November 2, 2013; accepted November 27, 2013

Copyright (C) 2013 Delfino Ladino-Luna et al. This is an open access article distributed under the Creative Commons Attribution License, which permits unrestricted use, distribution, and reproduction in any medium, provided the original work is properly cited.

\begin{abstract}
An analysis of the Stirling and Ericsson cycles from the point of view of the finite time thermodynamics is made by assuming the existence of internal irreversibilities in an engine modeled by these cycles, and the ideal gas as working substance is considered. Expressions of efficiency in both regimes maximum power output and maximum ecological function are also shown. Appropriate variables are introduced so that the objective functions, namely power output, ecological function and efficiency can be functions of the reservoirs temperatures ratio and certain "measurable" parameters as a thermal conductance, the general constant of gases and the compression ratio of the cycle. Several results from the finite time thermodynamics literature are used, so that the developed methodology leads directly to appropriate expressions of the objective functions in order to simplify the optimization process.
\end{abstract}

Keywords: Thermal Cycles; Efficiency; Power Output; Ecological Function

\section{Introduction}

As it is known, thermal engines can be assumed as endothermic or exothermic devices. Among the first, Otto and Diesel engines are the best known; and among the second two devices, Stirling and Ericsson engines are very interesting and similar to the theoretical Carnot engine $[1,2]$. They are engines closed-cycles regenerative devices initially used for various applications, particularly the Stirling cycle as water pumping and until the middle of last century manufactured on a large scale. But the development of the internal combustion engines from the mid-nineteenth century and the improvement in the refining of fossil fuels influenced the abandonment of the Stirling and the Ericsson engines in the race for industrialization, gradually since the early twentieth century. It is important to point out that Stirling and Ericsson cycles have an efficiency which goes towards the Carnot efficiency as it is shown in some textbooks.

It is also important to point out that a difference between endothermic and exothermic engines is the type of fuel used. While the first mentioned engines need fuel of a certain quality, the other engines can work with lowquality fuels and even alternative sources such as solar energy. Also in exothermic engines the working fluid does not change its composition during the cycle; these engines are quiet and safe but not very powerful. So, since the end of the previous century, and on recent times, Stirling and Ericsson engines characteristics have resulted in renewed interest in the study and design of such engines, and in the analysis of its theoretical idealized cycle, as it is shown in many papers [3-20]. Nevertheless, the discussion on these engines and its theoretical model has not been exhausted.

On other hand, the finite time thermodynamics theory actually is considered an extension of the classical equilibrium thermodynamics in the study of heat engines, in which explicitly is included time dependence of heat transfer processes between reservoirs and engine [21-28]. By excluding the irreversible processes occurring within the working substance, it is obtained the endoreversible cycle, but if the effects of these internal irreversible processes are took into account, the cycle is called non-endoreversible. The exclusion of such effects is known as endoreversibility hypothesis, and is considered for cases in which the internal relaxation time of the working substance is very small compared with the total cycle time. At first Curzon and Ahlborn analyzed the endoreversible Carnot cycle with finite heat transfer between hot and cold thermal reservoirs with temperatures $T_{H}$ and $T_{C}$, $T_{H}>T_{C}$, and the engine. Ideal gas as working substance 
operating at maximum power output was assumed [21]. The Carnot cycle was modified so that the working substance has $T_{H W}$ and $T_{C W}$ temperatures different than its reservoirs temperatures, fulfilling $T_{H}>T_{H W}>T_{C W}$ $>T_{C}$. The efficiency for this cycle is known as Curzon-Ahlborn-Novikov-Chambadal efficiency, and it is written by the definition $\varepsilon=T_{C} / T_{H}$ as [29,30],

$$
\eta_{C A N}=1-\sqrt{\varepsilon}
$$

In addition, as it is known, Carnot cycle in the classical equilibrium thermodynamics context is a cycle in which the working substance during the isothermal processes has the same temperature as the reservoirs, $T_{H}=T_{H W}$ and $T_{C W}=T_{C}$, with the efficiency,

$$
\eta_{C}=1-\varepsilon .
$$

References [8,11-20] are devoted to analysis of Stirling and Ericsson cycles in the finite time thermodynamics point of view. Particularly, with a similar analysis made by Curzon and Ahlborn [21], Angulo-Brown and Ramos-Madrigal analyzed these cycles [8], finding that its efficiency is towards efficiency given in (1).

On the other hand, it is known that real heat engines have internal processes that influence its performance. This is reason enough to consider an analysis of non endoreversible cycles. Several authors have addressed these problems as Ibrahim et al. [31], Chen [32], and Velasco et al. [33], among others, which analyzed the non-endoreversible Curzon and Ahlborn cycle.

Ibrahim et al. [31] showed the efficiency at maximum power of some cycles equal (or approximated to) $\eta_{m}=1-\sqrt{\varepsilon / \phi}$, where $\phi$ is a parameter to take into account internal irreversibilities in the cycle, with $0<\phi$ $\leq 1$. In the cycle without internal irreversibilities $\phi=1$. Chen [32] included this parameter as $I_{S} \equiv 1 / \phi$ and wrote the efficiency of a Curzon and Ahlborn cycle heat engine at maximum power given as $\eta_{m}=1-\sqrt{I_{S} \varepsilon}$, with $I_{S} \geq 1$. Velasco et al. [33] assumed the parameter $I \equiv 1 / I_{S}$ as a $\varepsilon$-independent parameter, and showed that $0.8 \leq I \leq 0.9$ for typical power plants with water as working fluid. Moreover, an adequate model of performance for modern plants is the ecological function, approached by Angulo-Brown [34], and in the maximum ecological function regime was found the efficiency $\eta_{E I}=1-\sqrt{\left(\varepsilon+\varepsilon^{2}\right) / 2 I}$ for the non-endoreversible Curzon and Ahlborn cycle [35].

Therefore, in the present paper, an analysis of the Stirling and Ericsson cycles from the viewpoint of finite time thermodynamics is now made. Assuming the existence of internal irreversibilities in an engine modeled by any of these cycles, it is shown modified expressions for the power output and ecological function, and examines how one can build the expression for the efficiency in both regimes, maximum power output and maximum eco- logical function. Some results from [8] are used, in order to obtain expressions for power output, ecological function and efficiency, similar to those obtained in [31,32, 35]. Variable changes are made, like those used in Gutkowicz-Krusin et al. [24], and Ladino-Luna [36]. This paper has two purposes; the first one is to show a methodology for writing objective functions including compression ratio as an important parameter; the second one is to show how in the context of finite time thermodynamics the Stirling and Ericsson cycles have an efficiency that in their limit cases is always reduced to the Curzon and Ahlborn cycle efficiency, as in classical equilibrium thermodynamics these cycles have an efficiency like the Carnot cycle efficiency.

\section{Stirling Cycle}

To construct expressions for power output and ecological function it is necessary make some initial assumptions. First, heat transfer of the process of the cycle is supposed to occur as Newton's cooling law for two bodies in thermal contact with temperatures $T_{i}$ and $T_{f}, T_{i}>T_{f}$, with a rapidity of heat change $\mathrm{d} Q / \mathrm{d} t$, and a constant thermal conductance $\alpha$, which for convenience is assumed to be equal in all cases of heat transfer,

$$
\frac{\mathrm{d} Q}{\mathrm{~d} t}=\alpha\left(T_{i}-T_{f}\right) \text {. }
$$

On the other hand, it is assumed that the internal processes of the system cause irreversibilities that can be represented by a factor $I_{S}$ [36], so from the second law of thermodynamics can be written

$$
Q_{C}=\frac{T_{C W}}{T_{H W}} I_{S} Q_{H}
$$

Power output is defined as,

$$
P=\frac{W_{t o t}}{t_{t o t}}
$$

where $W_{\text {tot }}=\sum_{j=1}^{4} W_{j}, W_{j}=$ work by the system in the $\mathrm{j}$-th process, $t_{t o t}=\sum_{i=1}^{4} t_{j}, t_{j}=$ time of $\mathrm{j}$-th process. Similarly the entropy production and the ecological function can be constructed taking into account (3) and (4), from its definition, i.e.,

$$
\sigma=\frac{\Delta S}{t_{\text {tot }}}
$$

and the ecological function also,

$$
E=P-T_{C} \sigma \text {. }
$$

As [24] shows, for an ideal gas as working substance in case of an isothermal process the equation of state leads to, 


$$
\frac{R T_{i}}{V} \frac{\mathrm{d} V}{\mathrm{~d} t}=\alpha\left(T_{i}-T_{f}\right) .
$$

Now, as it is known, Stirling cycle consists of two isochoric processes and two isothermal processes. At finite time is considered the difference between the temperatures of reservoirs and the corresponding operating temperatures, similarly to those of Curzon and Ahlborn cycle [21], as shown in Figure 1.

A fundamental assumption for this cycle is that the heating and cooling at constant volume are performed as,

$$
\left|\frac{\mathrm{d} T}{\mathrm{~d} t}\right|=r_{V}=\text { constant }
$$

where it is not dificult to show that it meets,

$$
\left|Q_{1 V}\right|=\left|Q_{2 V}\right| \text {. }
$$

On other hand, from the equilibrium conditions it can be assumed,

$$
\frac{\mathrm{d} U}{\mathrm{~d} t}=\frac{\mathrm{d} Q}{\mathrm{~d} t}=C_{V} \frac{\mathrm{d} T}{\mathrm{~d} t}=r_{V} C_{V},
$$

and since the heat of heating and cooling respectively, from the first law of thermodynamics,

$$
Q_{1 V}=C_{V}\left(T_{H W}-T_{C W}\right)=\Delta U_{41}
$$

and

$$
Q_{2 V}=C_{V}\left(T_{C W}-T_{H W}\right)=\Delta U_{23},
$$

and the time for each of isochoric processes are given as,

$$
t_{V}=\frac{1}{r_{V}}\left(T_{H W}-T_{C W}\right) \text {. }
$$

The time for the isothermal processes can be found from (8) as,

$$
t_{1}=\frac{R T_{H W}}{\alpha\left(T_{H}-T_{H W}\right)} \ln \frac{V_{2}}{V_{1}}
$$

and

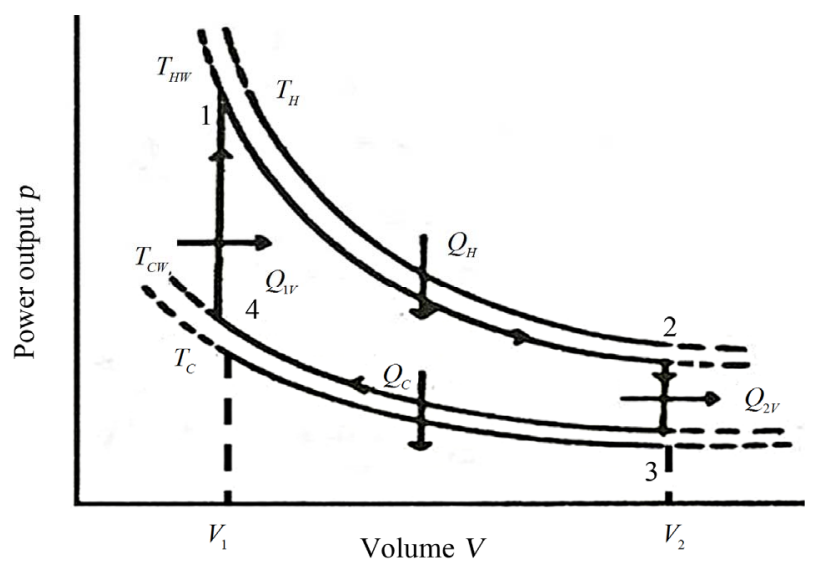

Figure 1. Idealized Stirling cycle at the $V-p$ (volume vs pressure) plane.

$$
t_{2}=-\frac{R T_{C W}}{\alpha\left(T_{C W}-T_{C}\right)} \ln \frac{V_{1}}{V_{2}},
$$

where the negative sign in (14b) is presented because there is no negative time, and the total time of the cycle is now,

$$
\begin{aligned}
t_{t o t}= & \frac{R T_{H W}}{\alpha\left(T_{H}-T_{H W}\right)} \ln \frac{V_{2}}{V_{1}}-\frac{R T_{C W}}{\alpha\left(T_{C W}-T_{C}\right)} \ln \frac{V_{1}}{V_{2}} \\
& +\frac{2}{r_{V}}\left(T_{H W}-T_{C W}\right) .
\end{aligned}
$$

So, since its definition and taking into account (4) power output of cycle is writen as,

$$
P=\frac{Q_{H}-I_{S} \frac{T_{C W}}{T_{H W}} Q_{H}}{t_{1}+t_{2}+2 t_{V}} .
$$

Now with the change of variables used in Ladino-Luna [35],

$$
Z_{I}=I_{S} \frac{T_{C W}}{T_{H W}} \text { and } u=\frac{T_{H W}}{T_{H}}
$$

and taking into account the ratio of temperatures of the heat reservoirs, used in (1) and (2), with the parameter $\lambda=\left[(\gamma-1) \ln \left(V_{2} / V_{1}\right)\right]^{-1}$ that includes the compression ratio of cycle $V_{2} / V_{1}=r_{C}$, the power output of Stirling cycle takes the form,

$$
P_{S I}=\frac{\alpha T_{H}\left(1-Z_{I}\right)}{\frac{1}{1-u}+\frac{I_{S} Z_{I}}{Z_{I} u-I_{S} \varepsilon}+\frac{2 \alpha T_{H}}{C_{V} I_{S} r_{V}}\left(I_{S}-Z_{I}\right) \lambda} .
$$

The optimization conditions $\left(\partial P_{S I} / \partial u\right)_{Z_{I}=\text { const }}=0$ and $\left(\partial P_{S I} / \partial Z_{I}\right)_{u=\text { const }}=0$ permit find the function $Z_{I}=Z_{I}\left(\varepsilon, I_{S}, \lambda\right)$. From the first one it is obtain $u=u\left(Z_{I}, I_{S}\right)$ as,

$$
u=\frac{\left(Z_{I}+\varepsilon \sqrt{I_{S}}\right) \sqrt{I_{S}}}{Z_{I}\left(1+\sqrt{I_{S}}\right)}
$$

and from the second one a solution physically adequate $Z_{I P}$ can be obtained by

$$
\begin{aligned}
& \frac{\left(1+\sqrt{I_{S}}\right)^{2} r_{V} C_{V} I_{S}+2 \alpha T_{H} \lambda\left(I_{S}-2 Z_{I}+\varepsilon I_{S}\right)}{-\left(Z_{I}-\varepsilon I_{S}\right)+\left(1-Z_{I}\right)} \\
& =\frac{Z_{I}\left(1+\sqrt{I_{S}}\right)^{2} r_{V} C_{V} I_{S}+2 \alpha T_{H} \lambda\left(Z_{I}-\varepsilon I_{S}\right)\left(I_{S}-Z_{I}\right)}{\left(1-Z_{I}\right)\left(Z_{I}-\varepsilon I_{S}\right)}
\end{aligned}
$$

so that the efficiency at maximum power output can be written as,

$$
\eta_{S I P}=1-Z_{I P}\left(\varepsilon, I_{S}, \lambda\right)
$$


for known values of parameters $C_{V}, \alpha$ and $T_{H}$. In the limit $\lambda \rightarrow 0$, namely $V_{2} / V_{1} \rightarrow \infty$, the efficiency of non-endoreversible Stirling cycle, $\eta_{S I P}$, goes to the efficiency for the non-endoreversible Curzon and Ahlborn cycle [33], as can be seen from (20),

$$
\eta_{S I P} \rightarrow \eta_{m}=1-\sqrt{\frac{\varepsilon}{I}} .
$$

The analysis for the case of ecological function is similar to the case of power output, and also leads to similar results. The shape of the function $u=u\left(Z_{I}, I_{S}, \varepsilon\right)$ is the same as in (19), but the form of $Z_{I}=Z_{I}\left(\varepsilon, I_{S}, \lambda\right)$ changes due to ecological function can be interpreted as an effective power output obtained of the heat engine. The simplest expression of ecological function is as (7) [34], where $T_{C}$ is the temperature of the cold reservoir and $\sigma$ is the entropy production definite as (6). Because heating and cooling in both isochoric and isobaric processes are considered constant, and taking into account (4) and (10), the change of entropy can be taken only for isothermal processes. Consequently the change of entropy for the non-endoreversible cycle considered is,

$$
\Delta S=-\frac{Q_{H}}{T_{H}}+\frac{Q_{C}}{T_{C}}=-\frac{Q_{H}}{T_{H}}+I_{S} \frac{T_{C W}}{T_{H W}} \frac{Q_{H}}{T_{C}},
$$

which leads to the ecological function as,

$$
\begin{aligned}
E & =\frac{Q_{H}}{t_{\text {tot }}}\left(1-2 I_{S} \frac{T_{C W}}{T_{H W}}+\frac{T_{C}}{T_{H}}\right) \\
& =\frac{R T_{H W}}{t_{\text {tot }}}\left(1-2 I_{S} \frac{T_{C W}}{T_{H W}}+\frac{T_{C}}{T_{H}}\right) \ln \frac{V_{2}}{V_{1}},
\end{aligned}
$$

where $t_{\text {tot }}$ is as (15). With the same parameters definite in (17) ecological function can be written now as,

$$
E_{S I}=\frac{\alpha T_{H}\left(1-2 Z_{I}+\varepsilon\right)}{\frac{1}{1-u}+\frac{I_{S} Z_{I}}{Z_{I} u-I_{S} \varepsilon}+\frac{2 \alpha T_{H}}{C_{V} I_{S} r_{V}}\left(I_{S}-Z_{I}\right) \lambda} .
$$

As in the case of power output optimizing, in order to find the efficiency at maximum ecological function there are also two conditions, namely $\left(\partial E_{S I} / \partial u\right)_{Z_{I}=\text { const }}=0$ and $\left(\partial E_{S I} / \partial Z_{I}\right)_{u=\text { const }}=0$. These conditions lead to obtaining the parameter $u$ as in (19) and also

$Z_{I E}=Z_{I E}\left(\varepsilon, I_{S}, \lambda\right)$ as an adequate solution for the second condition by the relation,

$$
\begin{aligned}
& \frac{Z_{I}\left(1+\sqrt{I_{S}}\right)^{2} r_{V} C_{V} I_{S}+2 T_{H} \alpha \lambda\left(Z_{I}-\varepsilon I_{S}\right)\left(I_{S}-Z_{I}\right)}{\left(1-2 Z_{I}+\varepsilon\right)\left(Z_{I}-\varepsilon I_{S}\right)} \\
& =\frac{\left(1+\sqrt{I_{S}}\right)^{2} r_{V} C_{V} I_{S}+2 T_{H} \alpha \lambda\left(I_{S}-2 Z_{I}+\varepsilon I_{S}\right)}{\left(1-2 Z_{I}+\varepsilon\right)-2\left(Z_{I}-\varepsilon I_{S}\right)}
\end{aligned}
$$

The efficiency for the Stirling cycle at maximum eco- logical function can be written now as,

$$
\eta_{\text {SIE }}=1-Z_{\text {IE }}\left(\varepsilon, I_{S}, \lambda\right),
$$

and also in the limit $\lambda \rightarrow 0$ goes towards the efficiency for non-endoreversible Curzon and Ahlborn cycle by (26), i.e.,

$$
\eta_{S I E} \rightarrow \eta_{E I}=1-\sqrt{\frac{\varepsilon+\varepsilon^{2}}{2 I}} .
$$

The existence of a finite heat transfer in the isothermal processes and the assumption of constant heating and cooling are affected with the assumption of a nonendoreversible cycle with ideal gas as working substance. However, the general form of power and ecological function of such analyzed cycles is similar to the corresponding previous expresions, obtained in the literature of finite time thermodynamics. One can see that $\lambda \rightarrow 0$ implies instantaneous adiabats in the case of Curzon and Ahlborn cycle, and the expresions for power output, ecological function and the corresponding eficiencies are the same as in Ladino-Luna [35,36].

Power output and ecological function have also an issue that shows direct dependence on the temperature of the working substance. Figures $\mathbf{2}$ and $\mathbf{3}$ show the approach of efficiencies obtained from (20) and (26) to the previously efficiencies found [31,32,35], for different values of compression ratio and assuming constant values to the parameters involved. In these figures can be appreciate that including besides the limit $I_{S} \rightarrow 0$ leads to the obtained expresions in Angulo-Brown and Ramos-Madrigal [8], but with the parameters used in the present paper.

Expressions obtained with the changes of variable suggested in (17) have the virtue of leading directly to the shape of the efficiency through the function $Z_{I}$. Figures 2 and $\mathbf{3}$ show as in classical equilibrium ther-

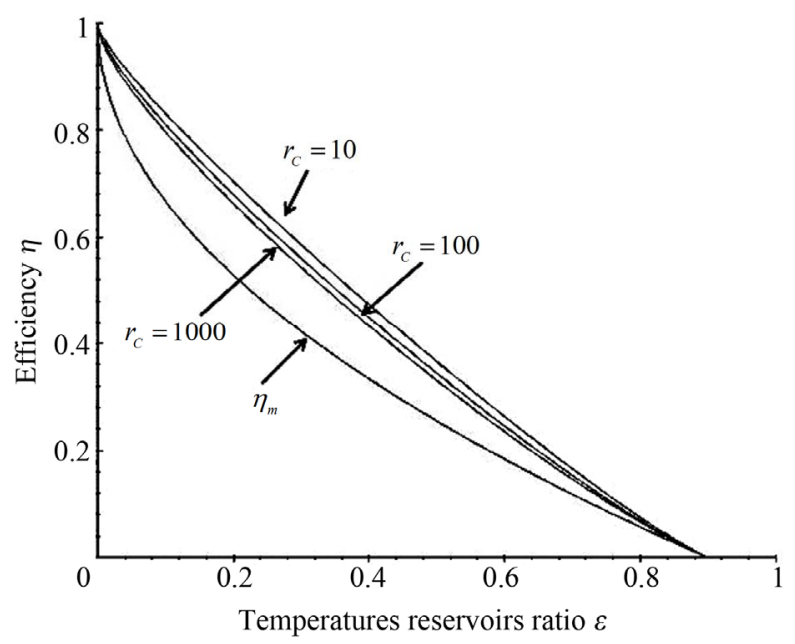

Figure 2. Comparison of resulting efficiencies from (20) with eficiencies in references [31,32]. 


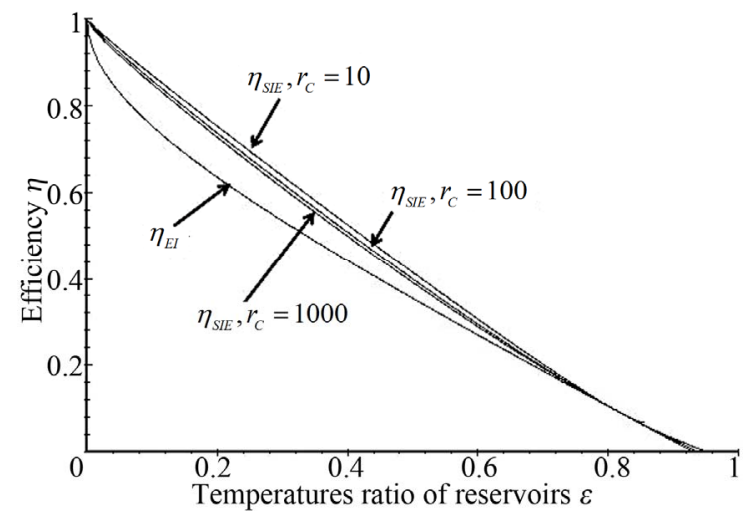

Figure 3. Comparison of resulting efficiencies from (26) with the efficiency in reference [35].

modynamics that the Stirling cycle has efficiency like the Carnot cycle efficiency, and how in the context of finite time thermodynamic this cycle has efficiency that in their limit cases is always reduced to the Curzon and Ahlborn cycle efficiency.

\section{Ericsson Cycle}

The Ericsson cycle, consisting of two isobaric processes and two isothermal processes is shown in Figure 4. Now, it is follows a similar procedure as in the Stirling cycle case.

The hypothesis on constant heating and cooling but now at constant pressure is expressed as,

$$
\left|\frac{\mathrm{d} T}{\mathrm{~d} t}\right|=r_{p}=\text { constant } .
$$

So is true that,

$$
\left|Q_{1 p}\right|=\left|Q_{2 p}\right|
$$

The equilibrium condition now is,

$$
\frac{\mathrm{d} U}{\mathrm{~d} t}=C_{p} \frac{\mathrm{d} T}{\mathrm{~d} t}-p \frac{\mathrm{d} V}{\mathrm{~d} t}=r_{p} C_{p}-p \frac{\mathrm{d} V}{\mathrm{~d} t},
$$

so the time for a constant pressure process is given as,

$$
t_{p}=\frac{T_{H W}}{r_{p}}\left(1-\frac{T_{C W}}{T_{H W}}\right) .
$$

and the time for the isothermal processes also can be obtained from (8), and can be written as,

$$
t_{1}=\frac{R T_{H W}}{\alpha\left(T_{H}-T_{H W}\right)} \ln \frac{V_{2}}{V_{1}}, t_{2}=-\frac{R T_{C W}}{\alpha\left(T_{C W}-T_{C}\right)} \ln \frac{V_{4}}{V_{3}}
$$

and the total time of cycle is now,

$$
\begin{aligned}
t_{\text {tot }}= & \frac{R T_{H W}}{\alpha\left(T_{H}-T_{H W}\right)} \ln \frac{V_{2}}{V_{1}}-\frac{R T_{C W}}{\alpha\left(T_{C W}-T_{C}\right)} \ln \frac{V_{4}}{V_{3}} \\
& +\frac{2}{r_{p}}\left(T_{H W}-T_{C W}\right),
\end{aligned}
$$

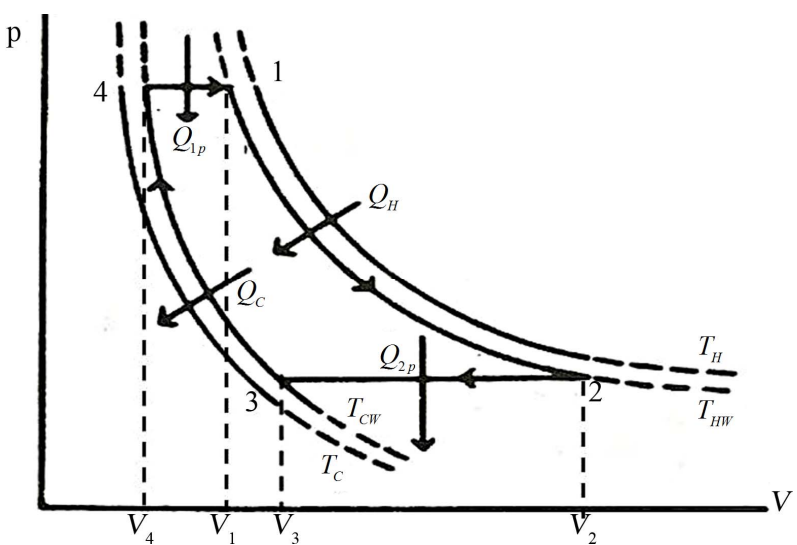

Figure 4. Idealized Ericsson cycle at the the $V-p$ (volume vs pressure) plane.

and the power output of cycle from its definition and taking into account (4) remains,

$$
P=\frac{Q_{H}-I_{S} \frac{T_{C W}}{T_{H W}} Q_{H}}{t_{1}+t_{2}+2 t_{p}} .
$$

With the change of variables suggested in (17) now the expression for the power output of non-endoreversible Ericsson cycle is,

$$
P_{E I}=\frac{\alpha T_{H}\left(1-Z_{I}\right)}{\frac{1}{1-u}+\frac{Z_{I}}{Z_{I} u-I_{S} \varepsilon}+\frac{2 \alpha T_{H}}{C_{V} I_{S} r_{p}}\left(I_{S}-Z_{I}\right) \lambda},
$$

which is essentially equal to that found for the Stirling cycle, with the only variation in the factor $r_{p}$ instead of $r_{v}$. For extreme conditions $\left(\partial P_{E I} / \partial u\right)_{u=\text { const }}=0$ and $\left(\partial P_{E I} / \partial u\right)_{Z_{I}=\text { const }}=0$, it is obtained again the expression (19), allowing us to find a physically acceptable solution $Z_{I P}=Z_{I P}\left(\varepsilon, I_{S}, \lambda\right)$ by,

$$
\begin{aligned}
& \frac{\left(1+\sqrt{I_{S}}\right)^{2} r_{p} C_{V} I_{S}+2 \alpha T_{H} \lambda\left(I_{S}-2 Z_{I}+\varepsilon I_{S}\right)}{-\left(Z_{I}-\varepsilon I_{S}\right)+\left(1-Z_{I}\right)} \\
& =\frac{Z_{I}\left(1+\sqrt{I_{S}}\right)^{2} r_{p} C_{V} I_{S}+2 \alpha T_{H} \lambda\left(Z_{I}-\varepsilon I_{S}\right)\left(I_{S}-Z_{I}\right)}{\left(1-Z_{I}\right)\left(Z_{I}-\varepsilon I_{S}\right)}
\end{aligned}
$$

So, at maximum power output condition the efficiency of non-endoreversible Ericsson cycle can be written as,

$$
\eta_{E I P}=1-Z_{I P}\left(\varepsilon, I_{S}, \lambda\right) .
$$

The analysis for the case of ecological function is similar to the case of power output, and also leads to similar results. The shape of the function $u=u\left(Z_{I}, I_{S}, \varepsilon\right)$ is the same as in (19), but the form of $Z_{I}=Z_{I}\left(\varepsilon, I_{S}, \lambda\right)$ changes due to ecological function can be interpreted as an effective power output obtained of the heat engine.

Again using the simplest expression of ecological function and because heating and cooling in isobaric proc- 
esses are considering constant, the change of entropy can be taken only for the isothermal processes. Consequently the change of entropy for the non-endoreversible Ericsson cycle considered is as,

$$
\Delta S=-\frac{Q_{H}}{T_{H}}+\frac{Q_{C}}{T_{C}}=-\frac{Q_{H}}{T_{H}}+I_{S} \frac{T_{C W}}{T_{H W}} \frac{Q_{H}}{T_{C}},
$$

from which the ecological function is,

$$
E=\frac{R T_{H W}}{t_{\text {tot }}}\left(1-2 I_{S} \frac{T_{C W}}{T_{H W}}+\frac{T_{C}}{T_{H}}\right) \ln \frac{V_{2}}{V_{1}},
$$

and substituting in (40) the total time (34), ecological function for the Ericsson cycle can be written as,

$$
E_{E I}=\frac{\alpha T_{H}\left(1-2 Z_{I}+\varepsilon\right)}{\frac{1}{1-u}+\frac{Z_{I}}{Z_{I} u-I_{S} \varepsilon}+\frac{2 \alpha T_{H}}{C_{V} I_{S} r_{p}}\left(I_{S}-Z_{I}\right) \lambda},
$$

where the parameter $r_{p}$ takes the adequate value depending on the cycle analyzed.

As in the case of power output optimizing, there are two conditions for the ecological efficiency at maximum ecological function, i.e, $\left(\partial E_{E I} / \partial u\right)_{z_{I}=\text { const }}=0$ and $\left(\partial E_{E I} / \partial Z_{I}\right)_{u=\text { const }}=0$. These conditions lead to obtain parameter $u$ as in (19), and also $Z_{E I}=Z_{E I}\left(\varepsilon, I_{S}, \lambda\right)$ by

$$
\begin{aligned}
& \frac{Z_{I}\left(1+\sqrt{I_{S}}\right)^{2} r_{p} C_{V} I_{S}+2 T_{H} \alpha \lambda\left(Z_{I}-\varepsilon I_{S}\right)\left(I_{S}-Z_{I}\right)}{\left(1-2 Z_{I}+\varepsilon\right)\left(Z_{I}-\varepsilon I_{S}\right)} \\
& =\frac{\left(1+\sqrt{I_{S}}\right)^{2} r_{p} C_{V} I_{S}+2 T_{H} \alpha \lambda\left(I_{S}-2 Z_{I}+\varepsilon I_{S}\right)}{\left(1-2 Z_{I}+\varepsilon\right)-2\left(Z_{I}-\varepsilon I_{S}\right)}
\end{aligned}
$$

The efficiency for Ericsson cycle at maximum ecological function can be written now as

$$
\eta_{\text {EIE }}=1-Z_{I E}\left(\varepsilon, I_{S}, \lambda\right) \text {. }
$$

\section{Concluding Remarks}

The developed methodology leads directly to appropriate expressions of the objective functions simplifying the optimization process, and this methodology shows the consequences of assuming non-endoreversible cyle in the process of isothermal heat transfer through the factor $I_{S}=1 / I$, which represents the internal irreversibilities of cycle [31], so that the proposed heat engine model is closer to a real engine. On other hand, as the known Carnot theorem provided a level of operation of heat engines, the Curzon and Ahlborn cycle provides levels of operation of such engines closer to reality. In this sense, the same manner within the context of classical equilibrium thermodynamics shows that in any cycle formed by two isothermal processes and any other pair of the same processes (isobaric, isochoric, adiabatic), efficiency tends to Carnot cycle efficiency, in the context of finite time thermodynamics, any cycle as previously mentioned has an efficiency which tends to Curzon and Ahlborn cycle efficiency. The above statements are independent if the cycle is considered endoreversible or non-endoreversible.

\section{Acknowledgements}

The authors thank the partial support of CONACYT (México) by the SNI program.

\section{REFERENCES}

[1] M. W. Zemansky and R. H. Dittman, "Heat and Thermodynamics," McGraw-Hill Book Company, Boston, 1968, Chap. 7.

[2] K. Wark, "Thermodynamics," McGraw-Hill Book Company, New York, 1983, Chap. 16.

[3] E. E. Daub, The British Journal for the History of Science, Vol. 7, 1974, pp. 269-257. http://dx.doi.org/10.1017/S0007087400013431

[4] T. K. O. Pettingil, "Thermodynamics of Stirling Type Engines for the Artificial Heart," Master Degree Thesis, McMaster University, Hamilton, 1977.

[5] J. R. Howell and R. B. Bannerot, Solar Energy, Vol. 19, 1977, pp. 149-153. http://dx.doi.org/10.1016/0038-092X(77)90052-4

[6] R. W. Satz, Energy Communications and Managements, Vol. 20, 1980, pp. 49-63. http://dx.doi.org/10.1016/0196-8904(80)90028-X

[7] H. Yayama and A. Tomokiyo, IEEE Transactions on Magnetics, Vol. MAG-23, 1987, pp. 2850-2852. http://dx.doi.org/10.1109/TMAG.1987.1065221

[8] F. Angulo-Brown and G. Ramos-Madrigal, Revista Mexicana de Física, Vol. 36, 1990, pp. 363-375.

[9] V. Bädescu, Energy, Vol. 17, 1992, pp. 601-607. http://dx.doi.org/10.1016/0360-5442(92)90095-H

[10] D. A. Blank and Ch. Wu, Energy Converse Management, Vol. 37, 1996, pp. 59-66. http://dx.doi.org/10.1016/0196-8904(95)00020-E

[11] J. Chen, Z. Yan, L. Chen and B. Andresen, International Journal of Energy Research, Vol. 22, 1998, pp. 805-812.

[12] D. A. Blank and Ch. Wu, Applied Thermal Engineering, Vol. 18, 1998, pp. 1347-1357. http://dx.doi.org/10.1016/S1359-4311(97)00080-X

[13] S. Ch. Kaushik, S. K. Tyagi, S. K. Bose and M. K. Singhal, International Journal of Thermal Sciences, Vol. 41, 2002, pp. 193-200. http://dx.doi.org/10.1016/S1290-0729(01)01297-2

[14] S. K. Tyagi, S. C. Kaushik and M. K. Singhal, Energy Convertion and Management, Vol. 43, 2002, pp. 22972309. http://dx.doi.org/10.1016/S0196-8904(01)00181-9

[15] S. K. Tyagi, S. C. Kaushik and R. Salohtra, Journal Physics D: Applied. Physics, Vol. 35, 2002, pp. 2058-2065. http://dx.doi.org/10.1088/0022-3727/35/16/323

[16] S. K. Tyagi, S. C. Kaushik and R. Salohtra, Journal Phy- 
sics D: Applied Physics, Vol. 35, 2002, pp. 2668-2675. http://dx.doi.org/10.1088/0022-3727/35/16/323

[17] B. Kongtragool and S. Wongwises, Renewable and Sustainable Energy Reviews, Vol. 7, 2003, pp. 131-154. http://dx.doi.org/10.1016/S1364-0321(02)00053-9

[18] S. K. Tyagi, G. Lin, S. C. Kaushik and J. Chen, International Journal of Refrigeration, Vol. 27, 2004, pp. 924931. http://dx.doi.org/10.1016/j.ijrefrig.2004.04.016

[19] U. Lucia, "Thermoeconomic Analysis of an Irreversible Stirling Heat Pump Cycle," 2005. arXiv:physics/0512182v1[physics.class-ph]

[20] I. Tlili, Renewable and Sustainable Energy Reviews, Vol. 16, 2012, pp. 2234-2241. http://dx.doi.org/10.1016/j.rser.2012.01.022

[21] F. L. Curzon and B. Ahlborn, American Journal Physics, Physics Review A, Vol. 15, 1976, pp. 2086-2093.

[22] B. Andresen, R. S. Berry, B. Nitzan and P. Salamon, Physics Review A, Vol. 15, 1976, pp. 2086-2093. http://dx.doi.org/10.1103/PhysRevA.15.2086

[23] P. Salamon, B. Andresen and R. S. Berry, Physics Review A, Vol. 15, 1976, pp. 2094-2101. http://dx.doi.org/10.1103/PhysRevA.15.2094

[24] D. Gutkowics-Krusin, I. Procaccia and J. Ross, Journal of Chemical Physics, Vol. 69, 1978, pp. 3898-3906. http://dx.doi.org/10.1063/1.437127

[25] M. Rubin, Physics Review A, Vol. 19, 1979, pp. 12721276. http://dx.doi.org/10.1103/PhysRevA.19.1272

[26] M. Rubin, Physics Review A, Vol. 19, 1979, pp. $1277-$ 1288. http://dx.doi.org/10.1103/PhysRevA.19.1277
[27] M. Rubin, Physics Review A, Vol. 22, 1980, pp. 17411752. http://dx.doi.org/10.1103/PhysRevA.22.1741

[28] P. Salamon and A. Nitzan, Physics Review A, Vol. 21, 1980, pp. 2115-2129. http://dx.doi.org/10.1103/PhysRevA.21.2115

[29] I. I. Novikov, Journal of Nuclear Energy II, Vol. 7, 1958, pp. 125-128.

[30] P. Chambadal, "Les Centrales Nucleares," Armand Colin Publisher, Paris, 1957, pp. 41-58.

[31] O. M. Ibrahim, S. A. Klein and J. W. Mitchell, Journal of Engineering for Gas Turbines and Power, Vol. 113, 1991, pp. 514-521. http://dx.doi.org/10.1115/1.2906271

[32] J. Chen, Journal Physics D: Applied Physics, Vol. 17, 1994, pp. 1144-1149. http://dx.doi.org/10.1088/0022-3727/27/6/011

[33] S. Velasco, J. M. M. Roco, A. Medina, J. A. White and A. Calvo-Hernández, Journal Physics D: Applied Physics, Vol. 33, 2000, pp. 355-359. http://dx.doi.org/10.1088/0022-3727/33/4/307

[34] F. Angulo-Brown, Journal Applied Physics, Vol. 69, 1991, pp. 7465-7469. http://dx.doi.org/10.1063/1.347562

[35] D. Ladino-Luna, Entropy, Vol. 9, 2005, pp. 186-197. http://dx.doi.org/10.3390/e9040186

[36] D. Ladino-Luna, Journal of the Energy Institute, Vol. 84, 2011, pp. 61-65. http://dx.doi.org/10.1179/014426011X12968328625315 